\title{
A globally convergent algorithm to compute all nash equilibria for $n$-person games
}

Citation for published version (APA):

Herings, P. J. J., \& Peeters, R. J. A. P. (2002). A globally convergent algorithm to compute all nash equilibria for n-person games. METEOR, Maastricht University School of Business and Economics. METEOR Research Memorandum No. 053 https://doi.org/10.26481/umamet.2002053

Document status and date:

Published: 01/01/2002

DOI:

10.26481/umamet.2002053

Document Version:

Publisher's PDF, also known as Version of record

\section{Please check the document version of this publication:}

- A submitted manuscript is the version of the article upon submission and before peer-review. There can be important differences between the submitted version and the official published version of record. People interested in the research are advised to contact the author for the final version of the publication, or visit the DOI to the publisher's website.

- The final author version and the galley proof are versions of the publication after peer review.

- The final published version features the final layout of the paper including the volume, issue and page numbers.

Link to publication

\footnotetext{
General rights rights.

- You may freely distribute the URL identifying the publication in the public portal. please follow below link for the End User Agreement:

www.umlib.nl/taverne-license

Take down policy

If you believe that this document breaches copyright please contact us at:

repository@maastrichtuniversity.nl

providing details and we will investigate your claim.
}

Copyright and moral rights for the publications made accessible in the public portal are retained by the authors and/or other copyright owners and it is a condition of accessing publications that users recognise and abide by the legal requirements associated with these

- Users may download and print one copy of any publication from the public portal for the purpose of private study or research.

- You may not further distribute the material or use it for any profit-making activity or commercial gain

If the publication is distributed under the terms of Article $25 \mathrm{fa}$ of the Dutch Copyright Act, indicated by the "Taverne" license above, 


\title{
A Globally Convergent Algorithm to Compute All Nash Equilibria for $n$-Person Games
}

\author{
P. Jean-Jacques Herings* Ronald Peeters*
}

December 10, 2002

\begin{abstract}
In this paper an algorithm is presented to compute all Nash equilibria for games in normal form on the only premises that the number of Nash equilibria is finite. The algorithm relies on decomposing the game by means of support-sets. For each support-set, the set of totally mixed equilibria of the support-restricted game that results by restricting the players to strategies in the support-set can be characterized by a system of polynomial equations and inequalities. By solving those systems for each support-set, all equilibria are found. The algorithm belongs to the class of homotopy-methods and is implementable. Finally, several techniques to speed up computations are proposed.
\end{abstract}

JEL Classification Codes: C63, C72.

Keywords: Computation of all equilibria; Non-cooperative game theory.

\section{Introduction}

In many research fields in which conflicts between agents arise, game-theoretic tools are used. In practical applications, game theory often has its shortcomings in fast computation of equilibria after a problem has been modeled as a game. This is the case in particular when games become very large, in the sense of many agents with many actions. One way to overcome this shortcoming lies in the development of algorithms to compute equilibria and select among equilibria. For many purposes, having an algorithm to compute a single sample equilibrium might be unsatisfactory. Even if the algorithm is able to select within

${ }^{*}$ Department of Economics, Universiteit Maastricht, P.O. Box 616, 6200 MD, Maastricht, The Netherlands (corresponding author: P. Jean-Jacques Herings, e-mail: P.Herings@algec.unimaas.nl).

We would like to thank Andy McLennan, T.E.S. Raghavan, Luc Habets and Anton Stoorvogel for their useful suggestions. The usual disclaimer applies. 
the complete set of equilibria an equilibrium that satisfies perfectness or some other refinement criterion, it cannot be neglected that there might exist another equilibrium that is more salient. For some refinements, for example risk dominance, a candidate equilibrium has to be compared with the other equilibria of the game. Even when an equilibrium for a game is found, there might exist multiple equilibria with different implications with regard to the original problem under consideration. All are motivations for having an algorithm to compute all equilibria.

For bimatrix games, efficient and implementable algorithms to compute all equilibria exist. For bimatrix games in which one player has exactly two strategies at its disposal, an algorithm to compute the complete set of Nash equilibria has been developed by Borm, Gijsberts, and Tijs (1987). For the general class of bimatrix games, algorithms have been developed by Dickhaut and Kaplan (1993) and Kostreva and Kinard (1991).

The algorithm that has been implemented in Gambit ${ }^{1}$ allows for finding all Nash equilibria of an $n$-person normal form game via the Liapunov function method described in McKelvey (1996). This is a continuously differentiable nonnegative function whose zeros coincide with the set of Nash equilibria of the game. A standard descent algorithm is used to find a constrained local minimum of the function from any given starting location. All global minima, which correspond to local minima with function value zero, are Nash equilibria of the game under consideration. These algorithms for computing 'all' equilibria will only find all equilibria (for generic games) in a weak probabilistic sense: Given any number less than one, there is an amount of time such that if the algorithm is run for at least that amount of time it will find all solutions with probability higher than that given number. For a general survey on the computation of equilibria, see McKelvey and McLennan (1996).

This paper presents a method that computes all Nash equilibria for finite $n$-person noncooperative games in normal form. The set of Nash equilibria can be represented as the set of solutions to a system of polynomial equations and inequalities. We decompose the system by means of all possible carrier structures, which makes the inequalities disappear. For the computation of the solutions to the resulting systems of polynomial equations, the homotopy approach is chosen. For homotopy continuation algorithms to solve systems of equations and inequalities of multivariate polynomials, a large library of literature is present.

As is well-known, see for instance McLennan (1999), the number of Nash equilibria increases exponentially in the size of the game. Therefore, the algorithm that is proposed

\footnotetext{
${ }^{1}$ Gambit is a library of game theory software and tools for the construction and analysis of finite extensive and normal form games. See http://www.hss.caltech. edu/ gambit/Gambit.html.
} 
is by definition exponentially. Exponential algorithms are often thought to be impractical. We do not completely share this view. The algorithm proposed here has the property that it generates more and more Nash equilibria during its execution, so there is no need to wait until it finally terminates. There is also the flexibility to start searching for particular equilibria, like Nash equilibria in pure strategies, or Nash equilibria in completely mixed strategies, before turning to others. Finally, it is possible to efficiently apply parallel computers to speed up computations.

This paper has been organized as follows. Some notations, definitions and general results are given in Section 2. In Section 3 a method to compute all equilibria is proposed. Section 4 deals in detail with the implementation of the proposed algorithm and proposes an explicit description of the algorithm. In Section 5 an alternative algorithm is presented in which Gröbner basis theory is applied. The proofs are collected in Section 6.

\section{Number of equilibria}

An $n$-person noncooperative game in normal form is a tuple $\Gamma=\left\langle N,\left\{S^{i}\right\}_{i \in N},\left\{u^{i}\right\}_{i \in N}\right\rangle$, with $N=\{1, \ldots, n\}$ the set of players, $S^{i}=\left\{s_{1}^{i}, \ldots, s_{\left|S^{i}\right|}^{i}\right\}$ the set of pure strategies of player $i$ and $u^{i}: S \rightarrow \mathbb{R}$ the payoff function of player $i$ which assigns to each pure strategy combination $s \in S=\chi_{i \in N} S^{i}$ a real number.

A mixed strategy of player $i$ is a probability distribution on $S^{i}$. Let $\Sigma^{i}$ denote the set of all probability distributions on $S^{i}$. For $\sigma^{i} \in \Sigma^{i}$, the probability assigned to pure strategy $s_{j}^{i}$ is given by $\sigma_{j}^{i}$. The strategy space of the game is therefore equal to $\Sigma=$ $\mathrm{X}_{i \in N} \Sigma^{i}$. Given a mixed strategy combination $\sigma \in \Sigma$ and a strategy $\bar{\sigma}^{i} \in \Sigma^{i}$, we denote by $\left(\sigma^{-i}, \bar{\sigma}^{i}\right)$ the mixed strategy that results from replacing $\sigma^{i}$ by $\bar{\sigma}^{i}$. If a mixed strategy combination $\sigma \in \Sigma$ is played, then the probability $\sigma(s)$ that the pure strategy combination $s=\left(s_{j^{1}}^{1}, \ldots, s_{j^{n}}^{n}\right)$ occurs is given by $\sigma(s)=\prod_{i \in N} \sigma_{j^{i}}^{i}$ and the expected payoff of player $i$ by $u^{i}(\sigma)=\sum_{s \in S} \sigma(s) u^{i}(s)$.

A mixed strategy combination $\sigma \in \Sigma$ is said to be a Nash equilibrium of the game $\Gamma$ if $\sigma^{i}$ is a best response against $\sigma^{-i}$ for all $i \in N$, i.e. there is no strategy $\bar{\sigma}^{i} \in \Sigma^{i}$ such that $u^{i}\left(\sigma^{-i}, \bar{\sigma}^{i}\right)>u^{i}(\sigma)$ for a certain player $i \in N$. The set of Nash equilibria of the game $\Gamma$ is denoted by $\mathrm{NE}(\Gamma)$ and is known to be non-empty.

From the existence of a Nash equilibrium, it is clear that for any game the number of equilibria is larger than or equal to one. It can also be shown that the number of Nash equilibria is generically finite and odd (see Harsanyi (1973), Rosenmüller (1971), and Wilson (1971)). Some more results are known on the number of equilibria of a normal form game with given size. For generic games, McKelvey and McLennan (1997) have determined 
the maximal number of totally mixed Nash equilibria and in McLennan (1997) the maximal number of pure Nash equilibria is determined. McLennan (1999) presents a formula for the expected number of Nash equilibria for a random normal form game for given (finite and non-empty) sets of players and pure strategies. In Table 1, given the number of players $(n)$ and the common number of strategies $(m)$, an estimation of the mean number of Nash equilibria is displayed with between brackets the standard error of the estimation.

\begin{tabular}{cccccc}
\hline$n \backslash m$ & 2 & 3 & 4 & 5 & 6 \\
\hline 2 & $1.31(0.13)$ & $1.52(0.12)$ & $1.77(0.18)$ & $2.64(0.44)$ & $2.61(0.52)$ \\
3 & $2.15(0.20)$ & $3.76(0.33)$ & $12.66(1.13)$ & $27.23(2.78)$ & $65.69(4.19)$ \\
4 & $4.49(0.40)$ & $18.01(1.22)$ & $82.49(3.87)$ & $440.02(18.86)$ & \\
5 & $6.98(0.43)$ & $81.82(3.32)$ & $879.24(32.94)$ & & \\
6 & $15.75(0.96)$ & $401.61(10.77)$ & & & \\
\hline
\end{tabular}

Table 1: Mean number of equilibria.

McLennan (1999) concludes from these numbers that the average number of Nash equilibria grows more rapidly than the size of the game, if the size of the game is measured by the number of pure strategy tuples times the number of players (which equals the number of payoffs in the game) and that the table clearly conveys the impression that the mean number of equilibria is greater when a large number of players each have a small number of pure strategies than the other way around.

\section{$3 \quad$ Equilibria as solutions to systems of equations}

In this section a method is presented to reformulate the problem of finding all Nash equilibria of a normal form game to finding all finite nonnegative real zeros of systems of multivariate polynomials. First a normal form game is decomposed by means of all possible carriers and secondly all totally mixed equilibria of the games that result by restricting the players to choose strategies within the predescribed carriers are computed.

The support-restricted games resulting from carriers with only two players having more than one strategy are equivalent to bimatrix games. For these support-restricted games existing algorithms developed for bimatrix games, such as for instance the algorithms proposed in Dickhaut and Kaplan (1993) and in Kostreva and Kinard (1991), can be applied and might improve computation time. Bimatrix games do only possess completely mixed equilibria if both players have an equal number of actions. This eliminates many 
carriers from consideration. Further, for each support-restricted game, there exists a 3person game such that the sets of completely mixed equilibria of both are isomorphic (see Bubelis (1979) and Datta (2002)). This result may be eventually useful for speeding up computations.

Let a subset $D^{*}$ of $S^{*}$ be given with the property that for every player $i$ there is at least one pure strategy $s_{j}^{i}$ in $D^{*}$, i.e. $D^{i}=D^{*} \cap S^{i} \neq \emptyset$ for every player $i$. Such a set $D^{*}$ is called admissible. Admissible subsets $D^{*}$ are used to decompose $\mathrm{NE}(\Gamma)$ in subsets $\mathrm{NE}\left(\Gamma, D^{*}\right)$, where $\operatorname{NE}\left(\Gamma, D^{*}\right)$ contains those elements of $\mathrm{NE}(\Gamma)$ where only strategies in $D^{*}$ are played with positive probability and all strategies in $D^{*}$ are best responses, i.e.

$$
\begin{aligned}
\mathrm{NE}\left(\Gamma, D^{*}\right)=\left\{\sigma \in \mathrm{NE}(\Gamma) \mid s_{j}^{i}\right. & \notin D^{*} \Rightarrow \sigma_{j}^{i}=0 \\
s_{j}^{i} & \left.\in D^{*} \Rightarrow s_{j}^{i} \in \operatorname{argmax}_{s_{\ell}^{i} \in S^{i}} u^{i}\left(\sigma^{-i}, s_{\ell}^{i}\right)\right\} .
\end{aligned}
$$

The situation where a Nash equilibrium $\sigma$ is an element of two different sets $\mathrm{NE}\left(\Gamma, D^{*}\right)$ is a knife-edge case. It can only occur if some player $i$ has an optimal strategy that is played with probability zero. It is easily seen that

$$
\mathrm{NE}(\Gamma)=\bigcup_{D^{*}} \mathrm{NE}\left(\Gamma, D^{*}\right) .
$$

An admissible subset $D^{*}$ of $S^{*}$ determines a support-restricted game $\Gamma_{\mid D^{*}}=\left\langle N,\left\{D^{i}\right\}_{i \in N},\left\{u^{i}\right\}_{i \in N}\right\rangle$ with $D^{i}=\left\{d_{1}^{i}, \ldots, d_{\left|D^{i}\right|}^{i}\right\}$ the set of pure strategies of player $i$ and the payoff functions restricted to the set $D=\chi_{i \in N} D^{i}$. A mixed strategy of player $i$ is a probability distribution on $D^{i}$. The set of mixed strategies for player $i$ will be denoted by $\Delta^{i}$, with generic element $\delta^{i}$, and we define $\Delta=\chi_{i \in N} \Delta^{i}$.

Given an admissible subset $D^{*}$, define

$$
\mathcal{E}\left(\Gamma_{\mid D^{*}}\right)=\left\{\delta \in \Delta \mid D^{i}=\operatorname{argmax}_{d_{\ell}^{i} \in D^{i}} u^{i}\left(\delta^{-i}, d_{\ell}^{i}\right) \quad \text { for all } i \in N\right\}
$$

as the set of all Nash equilibria $\delta$ of support-restricted game $\Gamma_{\mid D^{*}}$ with the property that for all players $i \in N$ it holds that all strategies from $D^{i}$ are best responses to $\delta^{-i}$.

If for an admissible subset $D^{*}$ it holds that a player has an action that is strictly dominated by an other action in the game $\Gamma_{\mid D^{*}}$ then $\mathcal{E}\left(\Gamma_{\mid D^{*}}\right)$ is empty. Furthermore, when for an admissible subset $D^{*}$ it holds that $\left(\left|D^{i}\right|-1\right)>\sum_{k \neq i}\left(\left|D^{k}\right|-1\right)$ for some $i$ then $\mathcal{E}\left(\Gamma_{\mid D^{*}}\right)$ is also empty (see McKelvey and McLennan (1997)). The intuition of the latter is that in order to be indifferent among all alternative actions, the opponents need enough strategic opportunities to make one indifferent among all alternative actions. Those carriers can be eliminated from consideration. 
For all $\delta \in \operatorname{NE}\left(\Gamma, D^{*}\right)$ it holds that $\delta \in \mathcal{E}\left(\Gamma_{\mid D^{*}}\right)$, i.e. $\operatorname{NE}\left(\Gamma, D^{*}\right) \subseteq \mathcal{E}\left(\Gamma_{\mid D^{*}}\right) .{ }^{2}$ Elements of $\mathcal{E}\left(\left.\Gamma\right|_{D^{*}}\right)$ are not necessarily elements of $\mathrm{NE}\left(\Gamma, D^{*}\right)$, since there may exist an $s^{i} \in S^{*} \backslash D^{*}$ with $u^{i}\left(\delta^{-i}, s^{i}\right)>u^{i}(\delta)$. Therefore it holds that $\operatorname{NE}\left(\Gamma, D^{*}\right)=\mathcal{E}\left(\Gamma_{\mid D^{*}}\right) \cap \operatorname{NE}(\Gamma)$.

If $\delta \in \mathcal{E}\left(\Gamma_{\mid D^{*}}\right)$, then

$$
\begin{aligned}
& u^{i}\left(\delta^{-i}, d_{j}^{i}\right)-u^{i}\left(\delta^{-i}, d_{\ell}^{i}\right)=0, \quad\left(d_{j}^{i}, d_{\ell}^{i} \in D^{i}, i \in N\right), \\
& \sum_{d_{j}^{i} \in D^{i}} \delta_{j}^{i}-1=0, \quad(i \in N) .
\end{aligned}
$$

The first line says that for each $\delta \in \mathcal{E}\left(\Gamma_{D^{*}}\right)$ all players $i \in N$ are indifferent between playing the pure strategies from $D^{i}$ when $\delta^{-i}$ is played by the opponents. The second line makes sure that the probabilities by which the players play certain strategies add up to one.

Fix one element $\tilde{d}^{i} \in D^{i}$ for each player $i$. This is possible because of the admissibility of the set $D^{*}$. Then the set of solutions to the set of equations above is equivalent to the set of solutions to the following system of multilinear equations:

$$
\begin{aligned}
& u^{i}\left(\delta^{-i}, \tilde{d}^{i}\right)-u^{i}\left(\delta^{-i}, d^{i}\right)=0, \quad\left(d^{i} \in D^{i} \backslash\left\{\tilde{d}^{i}\right\}, i \in N\right), \\
& \sum_{d_{j}^{i} \in D^{i}} \delta_{j}^{i}-1=0, \quad(i \in N) .
\end{aligned}
$$

All together, this system has $\sum_{i \in N}\left(\left|D^{i}\right|-1\right)+n=\left|D^{*}\right|$ equations and $\left|D^{*}\right|$ unknows. What one expects is a zero-dimensional solution set. To state this differently, one expects that the set of solutions to the system (1)-(2) consists of a finite number of isolated points. We proceed now by making this intuition more clear.

A normal form game can be parameterized by the payoffs; any game $\Gamma$ is determined by the set of players, number of actions per player, and some vector $u$ containing the payoffs of the game. When it is said that a property holds for almost every game $\Gamma$, it means that for any specification of the set of players and the number of pure strategies per player, the property holds for almost every vector $u$ that parameterizes this game $\Gamma$.

For every vector $u \in \mathbb{R}^{n|S|}$ and admissible subset $D^{*}$, define the function $F^{D^{*}, u}: \mathbb{R}^{\left|D^{*}\right|} \rightarrow$ $\mathbb{R}^{\left|D^{*}\right|}$ by the left-hand side of (1)-(2), i.e.

$$
F^{D^{*}, u}(\delta)=\left(\begin{array}{cc}
u^{i}\left(\delta^{-i}, \tilde{d}^{i}\right)-u^{i}\left(\delta^{-i}, d^{i}\right) & \left(d^{i} \in D^{i} \backslash\left\{\tilde{d}^{i}\right\}, i \in N\right) \\
\sum_{d_{j}^{i} \in D^{i}} \delta_{j}^{i}-1 & (i \in N)
\end{array}\right) .
$$

The set of solutions $\mathcal{E}\left(\Gamma_{\mid D^{*}}\right)$ to the system (1)-(2) is a subset of the set of solutions to $F^{D^{*}, u}(\delta)=0$. In fact, if $\delta \in \mathcal{E}\left(\Gamma_{\mid D^{*}}\right)$, then $\delta$ is a nonnegative real solution of $F^{D^{*}, u}(\delta)=0$.

\footnotetext{
${ }^{2}$ In fact, $\delta$ is not an element of $\Sigma$, but from $\Delta$. When we use a $\delta$ from $\Sigma$ we actually mean $\sigma(\delta)$ from $\Sigma$ where $\sigma(\delta)$ is the trivial extension of $\delta$ in $\Delta: \sigma_{j}^{i}(\delta)=\delta_{j}^{i}$ if $s_{j}^{i} \in D^{*}$ and $\sigma_{j}^{i}(\delta)=0$ otherwise.
} 
An element $\delta \in \mathbb{C}^{\left|D^{*}\right|}$, the set of $\left|D^{*}\right|$-dimensional complex vectors, is an element of $\mathcal{E}\left(\Gamma_{\mid D^{*}}\right)$ if it solves $(1)-(2)$ and

(a) $\quad \delta_{j}^{i} \in \mathbb{R}_{+}, \quad\left(d_{j}^{i} \in D^{i}, i \in N\right)$.

It follows that $\delta \in \mathbb{C}^{\left|D^{*}\right|}$ is an element of $\mathrm{NE}\left(\Gamma, D^{*}\right)$, and therefore a Nash equilibrium of the game $\Gamma$, if and only if it solves (1)-(2), (a), and

$$
u^{i}(\delta)-u^{i}\left(\delta^{-i}, s^{i}\right) \geq 0, \quad\left(s^{i} \in S^{i} \backslash D^{i}, i \in N\right) .
$$

Since (1) consists of $\left|D^{*}\right|-n$ polynomials of degree $n-1$ and (2) consists of $n$ polynomials of degree 1 , the total degree of the system $(1)-(2)$ is $(n-1)^{\left|D^{*}\right|-n}$ as being the product of the degrees of the individual equations. The theorem of Bezout says that the number of solutions and solutions at infinity, counting multiplicities, is equal to the total degree of the system (see Subsection 4.1).

Theorem 1 When the number of solutions in $\mathbb{C}^{D^{*} \mid}$ to $F^{D^{*}, u}=0$ is finite, it equals $(n-$ $1)^{\left|D^{*}\right|-n}$ (where solutions are counted by multiplicity and infinite solutions are counted).

The reason that the analysis is done in complex space is because the number of complex solutions to $F^{D^{*}, u}(\delta)=0$ is known when the solution set consists only of a finite number of isolated points. This enables us to make sure that all solutions have been found. While we are interested in finding all solutions to the system (1)-(2) that satisfy conditions $(a)$ (b), by finding all solutions with their multiplicities, one can be sure that all solutions to $F^{D^{*}, u}(\delta)=0$, or to (1)-(2), with the desired properties (Nash equilibria) have been found.

Although it is known that for a set of games with full Lebesgue measure the number of Nash equilibria is finite, it cannot be guaranteed that for the same set of games the set of (complex) solutions to $F^{D^{*}, u}(\delta)=0$ is finite for all admissible subsets $D^{*}$. In fact, up to now, there is no proof present that such a property holds. However, when the vector $u$ is allowed to be chosen from the complex space, the following holds true.

Theorem 2 For all admissible subsets $D^{*}$, there is a set of vectors $u \in \mathbb{C}^{n|D|}$ with full Lebesgue measure such that the set of solutions to $F^{D^{*}, u}(\delta)=0$ is a compact zero-dimensional manifold.

Proof See Section 6.

The proof of Theorem 2 is spelled out in Section 6 and exploits techniques as introduced in Herings (1997) and Herings and Peeters (2001). The set of vectors $u$ for which Theorem 2 holds for all admissible subsets equals the intersection of the separate sets over the admissible subsets. Since the number of admissible subsets is finite, it concerns a finite intersection. More precisely, it is a finite intersection of sets with full Lebesgue measure. 
Theorem 3 There is a set of vectors $u \in \mathbb{C}^{n|S|}$ with full Lebesgue measure such that for all admissible subsets $D^{*}$ the set of solutions to $F^{D^{*}, u}(\delta)=0$ is a compact zero-dimensional manifold.

Proof For an admissible subset $D^{*}$, let $\mathcal{U}\left(D^{*}\right)$ denote the set of full Lebesgue measure from Theorem 2. Define $\mathcal{U}=\bigcap_{D^{*}} \overline{\mathcal{U}}\left(D^{*}\right)$, where $\overline{\mathcal{U}}\left(D^{*}\right)$ is the class of vectors $u \in \mathbb{C}^{n|S|}$ for which the natural restriction to $\mathbb{C}^{n|D|}, u_{\mid D^{*}}$, is in $\mathcal{U}\left(D^{*}\right)$. That the set $\mathcal{U}$ is a set with full Lebesgue measure is obvious, as being a finite intersection of sets with full Lebesgue measure.

This latter result seems rather weak since payoffs are commonly in real numbers, and the set $\mathbb{R}^{n|S|}$ is a set of measure zero in $\mathbb{C}^{n|S|}$. However, when a game with payoff-vector $u$ in real numbers is considered which is not in the generic set of the theorem, a small perturbation of the payoff-vector (if necessary, complex) suffices to obtain a payoff-vector which is. For this newly obtained payoff-vector it is possible to compute all candidate equilibria. Since the equilibrium correspondence is upper hemi-continuous, the candidate equilibria found are close to - and therefore good approximations of - the equilibria for the original game defined by the payoff-vector $u$.

\section{The algorithm}

This section presents an algorithm to solve the systems of the previous section. The algorithm belongs to the class of homotopy-based algorithms. First, a general treatment of homotopy continuation methods to locate the zeros of a polynomial mapping is given. Next, one specific algorithm is discussed in detail, i.e. the algorithm used in the HoMPACKroutine POLSYS. Finally, a step-wise description of the algorithm is given.

\subsection{Homotopy continuation}

Many papers have been devoted to finding all solutions to a system $P$ of $n$ polynomial equations in $n$ unknowns using homotopy continuation ${ }^{3}$ methods on the only premises that the set of zeros is finite; see Chow, Mallet-Paret and Yorke (1979), Drexler (1977, 1978), Garcia and Li (1980), Garcia and Zangwill (1979a, 1979b, 1980), Kojima and Mizuno (1983), Mizuno (1981), Morgan (1983), Morgan and Sommese (1987), Morgan, Sommese and Watson (1989), Wright (1985), and Zulehner (1988).

\footnotetext{
${ }^{3}$ Homotopy continuation is also called imbedding, continuation or incremental loading.
} 
A map $P: \mathbb{C}^{n} \rightarrow \mathbb{C}^{n}$ is polynomial if the maps $P_{k}: \mathbb{C}^{n} \rightarrow \mathbb{C}$ are polynomials for all $k=1, \ldots, n$, i.e. $P_{k}(z)$ is a sum of terms each of which has the form $a z_{1}^{b_{1}} z_{2}^{b_{2}} \cdots z_{n}^{b_{n}}$ for some $a \in \mathbb{C}$ and some nonnegative integers $b_{j}(j=1, \ldots, n)$. The sum of all $b_{j}$ 's is the degree of the term, and the maximum of the degrees of the terms, $d_{k}$, is the degree of the polynomial $P_{k}$. The degree of $P$ is given by $d=\prod_{k=1}^{n} d_{k}$. Consider the system $P(z)=0$ of $n$ equations in $n$ unknowns. By Bezout's Theorem it follows that there are at most $d$ isolated solutions (if the number of solutions is finite).

Homotopy continuation methods can be used to find all the geometrically isolated solutions of $P(z)=0$. This works as follows. The system $P$ is embedded in a system of $n$ polynomial equations in $n+1$ unknowns where this new system includes the variables of $P$ and a new variable, the homotopy parameter. For one value of the homotopy parameter, the new system can be satisfactorily solved, and for another it is identical to $P$. The continuation process solves $P(z)=0$ by evolving or 'continuing' the full set of known solutions resulting for one value of the homotopy parameter into the full set of solutions to $P(z)=0$.

The homotopy system is denoted by $H(t, z)=0$, where $H(1, z)=P(z)$ for all $z$ and the solutions to $H(0, z)=0$ are known. The homotopy parameter $t$ varies between 0 and 1. The task is to follow the set of solutions to $H(t, z)=0$ that originate at $t=0$ and terminate at $t=1$. Assuming sufficient conditions so that $H^{-1}(\{0\})$ consists of smooth paths, the continuation towards the solutions becomes a process of path tracking.

Many issues arise in attempting to implement this concept into a reliable and fast algorithm for computing all solutions to polynomial systems. Basically, there are two steps:

(1) Define the homotopy $H(t, z)$.

(2) Choose a numerical method for tracking the paths defined by $H(t, z)=0$.

The definition chosen in step (1) has to result in smooth paths in $H^{-1}(\{0\})$ which link the known solutions of $H(0, z)=0$ to the solutions of $P(z)=0$. More precisely, the homotopy $H$ has to be chosen such that the components of $H^{-1}(\{0\})$ topologically have the following properties:

1. A component may be a closed arc which intersects each slice $\{t\} \times \mathbb{C}^{n}, t \in[0,1]$, once. These components correspond to single roots of the system $P(z)=0$.

2. A component may consist of $m$ arcs which meet in a single point of $\{1\} \times \mathbb{C}^{n}$. This point is a root of the system $P(z)=0$ with multiplicity $m$. Each slice $\{t\} \times \mathbb{C}^{n}$, $t \in[0,1)$, will intersect such a component in $m$ points. 
3. A component may be a half-open arc which intersects each slice $\{t\} \times \mathbb{C}^{n}, t \in[0,1)$, in a single point which tends to infinity as $t \rightarrow 1$. Such a component corresponds to an infinite root.

For the treatment of infinite roots there are two basic solutions: (a) define the homotopy such that for $t \in[0,1)$ the equations $H_{k}(t, z)=0$ are one degree higher than the equations $P_{k}(z)=0$, and (b) carry out the continuation in the complex projective space, a compactification of $\mathbb{C}^{n}$ which allows an explicit representation of infinite roots.

For step (2) there are two fundamental methods of numerically tracing those paths: predictor-corrector methods, and simplicial methods. Predictor-corrector methods approximately follow exact solution curves, whereas simplicial methods exactly follow approximate solution curves. For more theory on path-tracking methods the reader is referred to Allgower and Georg (1980, 1983, 1990, 1993) and Garcia and Zangwill (1981).

\subsection{Hompack}

Hompack (see Watson, Billups and Morgan (1987)) is a suite of codes that is programmed in FORTRAN and developed for following homotopy-paths numerically in order to compute fixed points or zeros. HOMPACK contains an algorithm, the POLSYS-routine, which allows to solve completely for systems of polynomial equations on the only premises that the solution set is finite. Separate routines are provided for dense and sparse matrices. In Morgan, Sommese and Watson (1989) it is described how the POLSYS routine of the software package HoMPACK computes all isolated solutions of a polynomial system.

Consider a polynomial map $P: \mathbb{C}^{n} \rightarrow \mathbb{C}^{n}$. We will take this polynomial map equal $F^{D^{*}, u}$ that results from the system of equations (1)-(2) given the choice of a specific carrier. Define $Q: \mathbb{C}^{n} \rightarrow \mathbb{C}^{n}$ by

$$
Q_{k}(z)=\beta_{k} z_{k}^{d_{k}}-\alpha_{k}, \quad k=1, \ldots, n,
$$

where $\alpha_{k}$ and $\beta_{k}$ are non-zero complex numbers, for $k=1, \ldots, n$. Define the homotopy $\operatorname{map} H_{\alpha, \beta}:[0,1] \times \mathbb{C}^{n} \rightarrow \mathbb{C}^{n}$ by

$$
H_{\alpha, \beta}(t, z)=(1-t) Q(z)+t P(z)
$$

where $\alpha=\left(\alpha_{1}, \ldots, \alpha_{n}\right) \in \mathbb{C}^{n}$ and $\beta=\left(\beta_{1}, \ldots, \beta_{n}\right) \in \mathbb{C}^{n}$. The following result of Morgan (1987, p. 124) applies.

Theorem 4 For any $P$, there are sets of measure zero, $A$ and $B$ in $\mathbb{C}^{n}$ such that, for $\alpha \notin A$ and $\beta \notin B$, the following holds: 
1. The solution set $\left\{(t, z) \in[0,1) \times \mathbb{C}^{n} \mid H_{\alpha, \beta}(t, z)=0\right\}$ is a collection of $d$ nonoverlapping (smooth) paths;

2. The paths move from $t=0$ to $t=1$ without backtracking in $t$;

3. Each geometrically isolated solution of $P(z)=0$ of multiplicity $m$ has exactly $m$ continuation paths converging to it;

4. A continuation path can diverge to infinity only as $t \rightarrow 1$;

5. If $P(z)=0$ has no solutions at infinity, all the paths remain bounded. If $P(z)=0$ has a solution at infinity, at least one path will diverge to infinity as $t \rightarrow 1$. Each geometrically isolated solution at infinity of $P(z)=0$ of multiplicity $m$ will generate exactly $m$ diverging continuation paths.

For almost all choices of $\alpha$ and $\beta$ in $\mathbb{C}^{n}, H_{\alpha, \beta}^{-1}(\{0\})$ consists of $d$ smooth paths emanating from $\{0\} \times \mathbb{C}^{n}$, which either diverge to infinity as $t$ approaches 1 or converge to a solution to $P(z)=0$ as $t$ approaches 1 . And, each geometrically isolated solution of $P(z)=0$ has a path converging to it.

Remark 1 From Theorem 3 it follows that for almost all games the polynomial map $F^{D^{*}, u}$ from Section 3 satisfies the properties required for the polynomial map P. Theorem 4 claims that with probability one the homotopy map $H$ defined above satisfies all nice properties needed for numerical path-tracking. Moreover, due to generic regularity of all zeroes of $F^{D^{*}, u}$ item 1. of Theorem 4 can be extended to hold true for all t's in the closed unit interval $[0,1]$ for a set of games with full Lebesgue measure.

In Hompack, the algebraic context for generating the full solution list of a polynomial system is complex projective space rather than real or complex Euclidean space, thereby immediately providing a treatment for the infinite roots. For HOMPACK therefore the classical approach from algebraic geometry of homogenizing $P$ and establishing the continuation process in projective space is followed.

The complex projective space, $\mathbb{C P}^{n}$, consists of the lines through the origin in $\mathbb{C}^{n+1}$, denoted $\left[\left(z_{0}, \ldots, z_{n}\right)\right]$, where $\left(z_{0}, \ldots, z_{n}\right) \in \mathbb{C}^{n+1} \backslash\{0\}$; that is, $\left[\left(z_{0}, \ldots, z_{n}\right)\right]$ is the line through the origin that contains $\left(z_{0}, \ldots, z_{n}\right)$. The complex projective space $\mathbb{C P}^{n}$ can be seen as the disjoint union of points $\left[\left(z_{0}, \ldots, z_{n}\right)\right]$ with $z_{0} \neq 0$ identified with the Euclidean space via $\left[\left(z_{0}, \ldots, z_{n}\right)\right] \rightarrow\left(z_{1} / z_{0}, \ldots, z_{n} / z_{0}\right)$ and the 'points at infinity,' the elements $\left[\left(z_{0}, \ldots, z_{n}\right)\right]$ with $z_{0}=0$. 
Given $P_{k}\left(z_{1}, \ldots, z_{n}\right)$, let $P_{k}^{\perp}\left(z_{0}, \ldots, z_{n}\right)$ be defined as follows. Each term of $P_{k}^{\perp}$ is obtained from the corresponding term of $P_{k}$ by multiplying it by the power of $z_{0}$ to bring the degree of the term up to $d_{k}$. Thus, a term of $P_{k}$ of degree $\delta$ is multiplied by $z_{0}^{d_{k}-\delta}$, and consequently each term of $P_{k}^{\perp}$ has degree $d_{k}$. Thus, $P_{k}^{\perp}(\lambda z)=\lambda^{d_{k}} P_{k}^{\perp}(z)$, and $P_{k}^{\perp}$ takes all points of $\left[\left(z_{0}, \ldots, z_{n}\right)\right]$ to the same point. The map $P_{k}^{\perp}: \mathbb{C P}^{n} \rightarrow \mathbb{C}$ is the homogenization of the map $P_{k}$. Then, $P^{\perp}$ - all $n$ components $P_{k}^{\perp}$ taken together - is a map form $\mathbb{C P}^{n}$ to $\mathbb{C}^{n}$. Note that if $P^{\perp}(z)=0$, then $P^{\perp}(\lambda z)=0$, for any non-zero complex scalar $\lambda$. Therefore, 'solutions' of $P^{\perp}(z)=0$ are (complex) lines through the origin.

The system $P^{\perp}\left(z_{0}, \ldots, z_{n}\right)=0$ reduces to the system $P(z)=0$ under the substitution $z_{0}=1$. Thus, the two systems can be considered to have the same set of roots in $\mathbb{C}^{n}$.

Theorem 5 There are no more than $d$ isolated solutions to $P^{\perp}(y)=0$ in $\mathbb{C P}^{n}$. If $P^{\perp}(y)=0$ has only a finite number of solutions in $\mathbb{C P}^{n}$, it has exactly d solutions, counting multiplicities.

To avoid dealing with $\mathbb{C P}^{n}$, a unique point is determined for each solution line. This point is $z \in \mathbb{C}^{n}$ such that either $(1, z)$ is on the solution line, or $(0, z)$ is and the first non-zero component of $z$ is 1 .

\subsection{Description of the algorithm}

In this subsection the proposed algorithm to compute all equilibria is comprehensively described in a step-wise manner.

Step 1. In the first step of the algorithm, the game is decomposed by means of supportsets. Each decomposition can be seen as a game for which the strategy set of each player is restricted as only to use strategies that belong to the support-set.

Step 2. If for the support-restricted game one player has a strictly dominated strategy or one player has too many actions relative to the other players, $\left(\left|D^{i}\right|-1>\sum_{k \neq i}\left|D^{k}\right|-1\right)$ for some $i$, see McKelvey and McLennan (1997), then it can be concluded that this supportrestricted game does not possess a totally mixed Nash equilibrium. In such a case, no more computations are needed. When the support-restricted game has a special structure-in particular, when only two players have more than one strategy such that the supportrestricted game is equivalent to a bimatrix game - it is possible to use existing algorithms especially developed for the class of games having that structure. In this case we can forward the found candidate equilibria to the final step of the algorithm. Otherwise a set of polynomial equations is formulated for which the set of solutions contain all completely mixed equilibria of the support-restricted game. 
Step 3. In this step the bulk of the computations are done by using a numerical method to compute all solutions of the system of polynomial equations according to (1)-(4). The POLSYS-routine of HOMPACK is used to do these computations. The found candidate equilibria are exported to the next step.

Step 4. The candidate equilibria found are checked whether they really are equilibria of the original game. The solutions that are not nonnegative and real are removed as are the solutions for which there is a player that can obtain a better payoff by using a pure strategy that is outside the support-set.

\section{An alternative algorithm}

This section presents an alternative method to solve the systems of the Section 3 using Gröbner basis theory. In the first subsection, the Gröbner basis is presented comprehensively. The second subsection deals with the application of the Gröbner basis to normal form games.

\subsection{The Gröbner basis}

Let $\mathbb{C}\left[z_{1}, \ldots, z_{n}\right]$ denote the set of all polynomials in $n$ variables with coefficients in $\mathbb{C}$. For $p_{1}, \ldots, p_{s} \in \mathbb{C}\left[z_{1}, \ldots, z_{n}\right]$, the variety $V\left(p_{1}, \ldots, p_{s}\right)$ is defined to be the set of solutions of the system

$$
p_{1}=0, \ldots, p_{s}=0
$$

That is,

$$
V\left(p_{1}, \ldots, p_{s}\right)=\left\{\left(a_{1}, \ldots, a_{n}\right) \in \mathbb{C}^{n} \mid p_{i}\left(a_{1}, \ldots, a_{n}\right)=0, i=1, \ldots, s\right\} .
$$

The set $I=\left\langle p_{1}, \ldots, p_{s}\right\rangle=\left\{\sum_{i=1}^{s} u_{i} p_{i} \mid u_{i} \in \mathbb{C}\left[z_{1}, \ldots, z_{n}\right], i=1, \ldots, s\right\}$ is an ideal in $\mathbb{C}\left[z_{1}, \ldots, z_{n}\right]$; that is, if $p, q \in I$, then so is $p+q$ and if $p \in I$ and $r$ is any polynomial in $\mathbb{C}\left[z_{1}, \ldots, z_{n}\right]$, then $r p \in I$. The set $\left\{p_{1}, \ldots, p_{s}\right\}$ is called a generating set of the ideal $I$. According to Adams and Loustaunau (1994, p.3) the following holds.

Theorem 6 The variety $V(I):=\left\{\left(a_{1}, \ldots, a_{n}\right) \in \mathbb{C}^{n} \mid p\left(a_{1}, \ldots, a_{n}\right)=0, p \in I\right\}$ is equal to the variety $V\left(p_{1}, \ldots, p_{s}\right)$. Or, stated differently, $p=0(p \in I)$ is equivalent to $p_{1}=$ $0, \ldots, p_{s}=0$.

Now, if we have $I=\left\langle p_{1}, \ldots, p_{s}\right\rangle=\left\langle p_{1}^{\prime}, \ldots, p_{t}^{\prime}\right\rangle$, then $V\left(p_{1}, \ldots, p_{s}\right)=V(I)=V\left(p_{1}^{\prime}, \ldots, p_{t}^{\prime}\right)$. This means that the system $p_{1}=0, \ldots, p_{s}=0$ has the same solutions as the system 
$p_{1}^{\prime}=0, \ldots, p_{t}^{\prime}=0$, and hence a variety is determined by an ideal, not by a particular set of equations. If we have a 'better' generating set for the ideal $I=\left\langle p_{1}, \ldots, p_{s}\right\rangle$, we will have a 'better' representation for the variety $V\left(p_{1}, \ldots, p_{s}\right)$. By 'better' is meant a set of generators that allows us to understand the algebraic structure of $I=\left\langle p_{1}, \ldots, p_{s}\right\rangle$ and the geometric structure of $V\left(p_{1}, \ldots, p_{s}\right)$ better. This 'better' generating set for $I$ is called a Gröbner basis for $I$ (see Gröbner $(1949,1970)$ ). In the case of linear polynomials this 'better' generating set is the one obtained from the row echelon form of the matrix in the system.

Applying Buchberger's algorithm, see Buchberger (1965), to a zero-dimensional ideal $I,{ }^{4}$ a typical Gröbner basis can be found, namely one in 'triangular' form (this is similar to the row echelon form in the linear case). Thus, in order to solve the system of equations determined by a zero-dimensional ideal $I$, it suffices to have an algorithm to find the roots of polynomials in one variable. That is, first the equation in one variable, $q_{1}\left(z_{1}\right)=0$, is solved. Subsequently, for each solution $\alpha$ of $q_{1}\left(z_{1}\right)=0$, the equation $q_{2}\left(\alpha, z_{2}\right)=0$ is solved. The computation process continues in this manner all the way until $q_{n}\left(\alpha_{1}, \ldots, \alpha_{n-1}, z_{n}\right)=0$ is solved. The solutions obtained in this way are the only possible solutions. Finally the solutions are tested by equations $q_{n+1}=0, \ldots, q_{t}=0$ (in the case when $t>n$ ) in order to obtain the set of solutions of the full system of equations. For a thorough introduction into Gröbner bases, the reader is referred to Adams and Loustaunau (1994) and Cox, Little, and O'Shea (1996).

\subsection{Applying the Gröbner basis}

In the third step of the algorithm proposed in Section 4, HompaCK was used to compute all solutions of the system of polynomial equations. In this subsection it is shown how this third step can be altered by applying Gröbner basis theory instead of using HomPACK. Using the Gröbner basis the problem of solving a system of multivariate polynomials is transformed to the problem of (subsequently) solving single polynomial equations with one unknown. For the deformation of the system of polynomial equations into one in 'triangular' form, Buchberger's algorithm can be used.

An important advantage of having the triangular structure is that the problem of finding all zeros of a system of polynomials can be reduced to repeatedly finding all zeros of a single polynomial. Each step the zeros that are not finite, nonnegative and real can be filtered out, which increases performance of the algorithm in terms of computation time.

\footnotetext{
${ }^{4}$ Since the field of concern is the complex space and given the fact that this space is algebraically closed, saying that the ideal $I$ is zero-dimensional is equivalent to saying that the variety $V(I)$ is finite.
} 
A disadvantage of the application of the Gröbner basis is that the degree of the 'new' system may increase enormously, since the number of polynomials as well as the degree of each polynomial separately may increase. However, all polynomials except the first one to solve are linear with probability one as the following theorem shows.

Theorem 7 There is a set of vectors $u \in \mathbb{C}^{n|S|}$ with full Lebesgue measure such that for all admissible subsets $D^{*}$ the Gröbner basis of $F^{D^{*}, u}$ is a system of polynomials that are all linear, except one.

Proof See Section 6.

\section{Proofs}

To make the proofs as transparent as possible, we need some notations and definitions.

For $k \in \mathbb{Z}_{+}$, for $r \in \mathbb{N}^{*}$, a subset $X$ of $\mathbb{C}^{m}$ is called a $k$-dimensional $C^{r}$ manifold if for every $\bar{x} \in X$ there exists a local $C^{r}$ coordinate system of $\mathbb{C}^{m}$ around $\bar{x}$, i.e. a $C^{r}$ diffeomorphism $\phi: U \rightarrow V$, where $U$ is an open subset of $\mathbb{C}^{m}$ containing $\bar{x}$ and $V$ is open in $\mathbb{C}^{m}$, such that $\phi(\bar{x})=0$ and $\phi(X \cap U)=\left\{y \in V \mid y_{i}=0,(i=1, \ldots, m-k)\right\}$.

A way to obtain manifolds is by means of regular constraint sets. In general, a regular constraint set is a system of equalities and inequalities. Here, it is sufficient to restrict ourselves to systems of equalities only. Let $J$ be a finite index sets and let $\tilde{g}_{j}$ for all $j \in J$ be $C^{r}$ functions defined on some open subset $U$ of $\mathbb{C}^{m}$. Define

$$
M[\tilde{g}]=\left\{x \in U \mid \tilde{g}_{j}(x)=0, \forall j \in J\right\} .
$$

If for every $\bar{x} \in M[\tilde{g}]$ it holds that

$$
\left\{\partial_{x} \tilde{g}_{j}(\bar{x}) \mid j \in J\right\}
$$

is a set of independent vectors, then $M[\tilde{g}]$ is called a $C^{r}$ regular constraint set (RCS). In Jongen, Jonker and Twilt (1983) it is shown that every $C^{r}$ RCS is an $(m-|J|)$-dimensional $C^{r}$ manifold.

To prove Theorem 2, the following lemma is needed.

Lemma 8 For all admissible subsets $D^{*}$, there is a set of vectors $u \in \mathbb{C}^{n|S|}$ with full Lebesgue measure such that the Jacobian of $F^{D^{*}, u}$ has full rank in its zero points.

Proof Let an admissible subset $D^{*}$ of $S^{*}$ and a vector $u \in \mathbb{C}^{n|S|}$ be given and let the function $F^{D^{*}}: \mathbb{C}^{\left|D^{*}\right|} \times \mathbb{C}^{n|D|} \rightarrow \mathbb{C}^{\left|D^{*}\right|}$ be defined such that $F^{D^{*}}(\delta, u)=F^{D^{*}, u}(\delta)$. If the 
Jacobian of $F^{D^{*}}$ evaluated at $(\bar{\delta}, \bar{u}), \partial_{(\delta, u)} F^{D^{*}}(\bar{\delta}, \bar{u})$, has full rank for all $(\bar{\delta}, \bar{u})$ such that $F^{D^{*}}(\bar{\delta}, \bar{u})=0$, then it follows by the transversality theorem (see, for example, Mas-Colell (1985), Theorem I.2.2) that $\partial_{\delta} F^{D^{*}, u}(\bar{\delta})$ has full rank for all $\bar{\delta}$ such that $F^{D^{*}, u}(\bar{\delta})=0$, except for a set of vectors $u$ with zero Lebesgue measure. So, we have to prove that $\partial_{(\delta, u)} F^{D^{*}}$ has full rank in points $(\bar{\delta}, \bar{u})$ such that $F^{D^{*}}(\bar{\delta}, \bar{u})=0$.

It is easily seen that

$$
\begin{aligned}
& \partial_{\delta_{\ell}^{k}}\left(\sum_{d_{j}^{i} \in D^{i}} \delta_{j}^{i}-1\right)=\mathbb{1}_{i=k}, \\
& \partial_{u^{k}\left(\underline{\boldsymbol{d}}^{-k}, d_{\ell}^{k}\right)}\left(\sum_{d_{j}^{i} \in D^{i}} \delta_{j}^{i}-1\right)=0,
\end{aligned}
$$

where $\mathbb{1}$ represents the identity function that assigns value one if the condition in the subscript is satisfied and zero otherwise, and that

$$
\begin{aligned}
& \partial_{\delta_{\ell}^{k}}\left(u^{i}\left(\delta^{-i}, \tilde{d}^{i}\right)-u^{i}\left(\delta^{-i}, d_{j}^{i}\right)\right)=\left(u^{i}\left(\delta^{-i, k}, d_{\ell}^{k}, \tilde{d}^{i}\right)-u^{i}\left(\delta^{-i, k}, d_{\ell}^{k}, d_{j}^{i}\right)\right) \cdot \mathbb{1}_{i \neq k}, \\
& \partial_{u^{k}\left(\underline{d}^{-k}, d_{\ell}^{k}\right)}\left(u^{i}\left(\delta^{-i}, \tilde{d}^{i}\right)-u^{i}\left(\delta^{-i}, d_{j}^{i}\right)\right)=\delta\left(\underline{d}^{-i}\right) \cdot\left(\mathbb{1}_{d_{\ell}^{k}=\tilde{d}^{i}}-\mathbb{1}_{d_{\ell}^{k}=d_{j}^{i}}\right) .
\end{aligned}
$$

Further we know that

$$
\sum_{d^{-i} \in D^{-i}} \delta\left(d^{-i}\right)=1
$$

and therefore

$$
\sum_{d^{-i} \in D^{-i}} \partial_{u^{k}\left(d^{-k}, d_{\ell}^{k}\right)}\left(u^{i}\left(\delta^{-i}, \tilde{d}^{i}\right)-u^{i}\left(\delta^{-i}, d_{j}^{i}\right)\right)=\mathbb{1}_{d_{\ell}^{k}=\tilde{d}^{i}}-\mathbb{1}_{d_{\ell}^{k}=d_{j}^{i}} \cdot
$$

So, the Jacobian of $F^{D^{*}}$ in points $(\bar{\delta}, \bar{u})$ for which it holds that $F^{D^{*}}(\bar{\delta}, \bar{u})=0$ has the form as depicted in Figure 1 (for a certain ordering of variables and equations). Note that all derivatives are with respect to complex variables. In this figure the box containing the star is not specified because it is not necessary to know that part of the matrix to conclude that the matrix has full rank.

Proof of Theorem 2 From Lemma 8, it follows that for any admissible subset $D^{*}$ and for almost every vector $u$, the set $\left\{\delta \in \mathbb{C}^{\left|D^{*}\right|} \mid F^{D^{*}, u}(\delta)=0\right\}$ is a regular constraint set. By counting the number of equations and variables it follows that $\left\{\delta \in \mathbb{C}^{\left|D^{*}\right|} \mid F^{D^{*}, u}(\delta)=0\right\}$ is a zero-dimensional manifold.

Since $F^{D^{*}, u}$ is polynomial, the solution to $F^{D^{*}, u}=0$ is a semi-algebraic set. It is a wellknown result in semi-algebraic theory, see for instance Bochnac, Coste and Roy (1987), that the solution set has a finite number of components. A zero-dimensional manifold with a finite number of components is compact.

Proof of Theorem 7 Suppose to the contrary that for some game $\Gamma$ and some admissible 


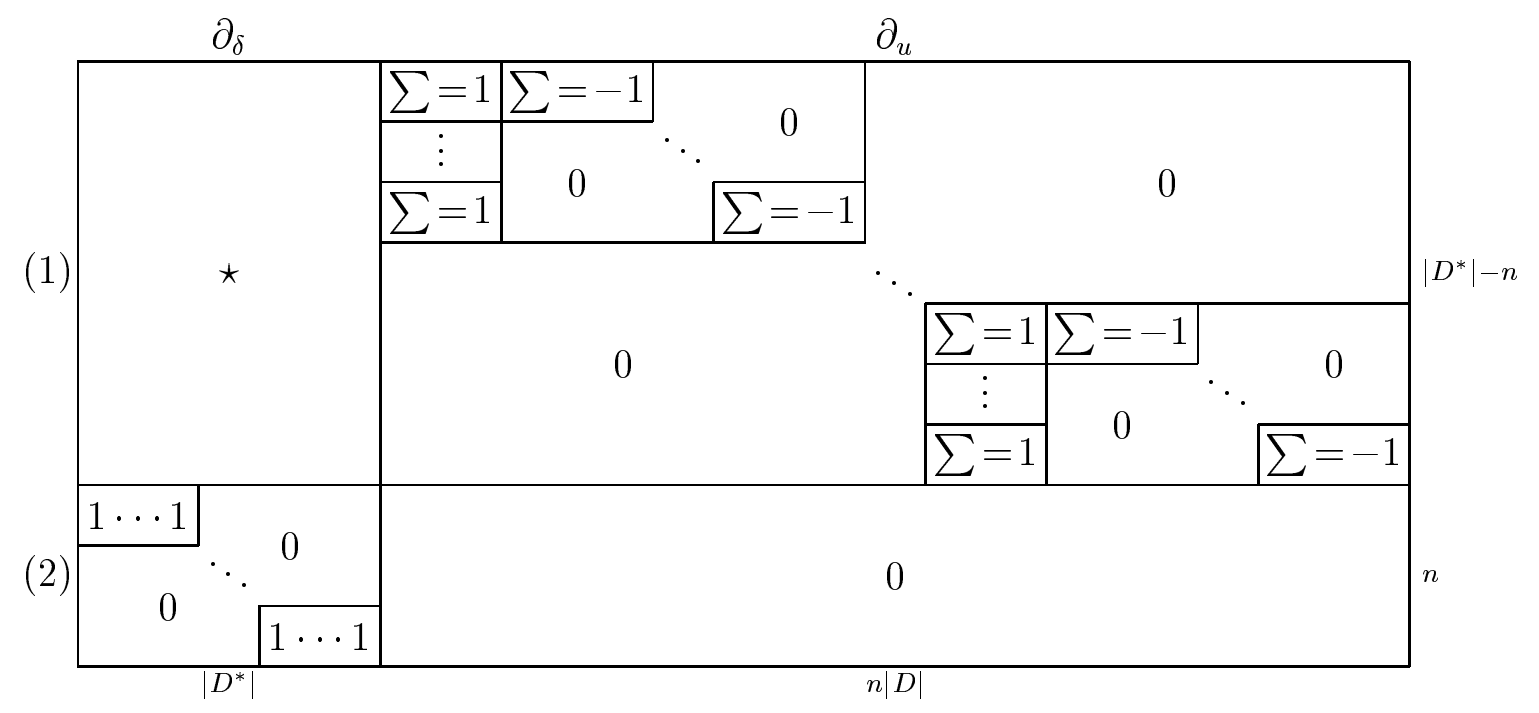

Figure 1: Jacobian of $F^{D^{*}}$.

subset $D^{*}$, the Gröbner basis of $F^{D^{*}, u(\Gamma)}$ has two or more polynomials that are not linear. Since such a polynomial has at least two solutions, it follows that there are two solutions, $\delta$ and $\gamma$, in which a single player $i$ plays one action $d_{j}^{i}$ with the same probability, i.e. $\delta_{j}^{i}=\gamma_{j}^{i}$. Moreover, player $i$ can be chosen such that he has at least two strategies at its disposal. Indeed, otherwise the polynomial that determines the probability of his pure strategy has to be linear, specifying the probability to be equal to one. We will show that this is impossible by constructing a class of games with full Lebesgue measure that satisfies certain transversality conditions. Next we show these transversality conditions to be incompatible with the existence of multiple solutions, where one action is played with the same probability.

Fix a carrier $D^{*}$, two different players $i^{1}$ and $i^{2}$, a strategy $j^{1}$ for player $i^{1}$, and a strategy $j^{2}$ for player $i^{2}$. Define the set $\tilde{\Delta}=\left\{(\delta, \gamma) \in \mathbb{C}^{\mid D^{*}}\left|\times \mathbb{C}^{\left|D^{*}\right|}\right| \delta_{j^{1}}^{i^{1}} \neq \gamma_{j^{1}}^{i^{1}}, \delta_{j^{2}}^{i^{2}} \neq\right.$ $\gamma_{j^{2}}^{i^{2}}$. Moreover, fix a player $i^{3}$ and a strategy of that player, $j^{3}$, where $\left(i^{3}, j^{3}\right) \neq\left(i^{1}, j^{1}\right)$, $\left(i^{3}, j^{3}\right) \neq\left(i^{2}, j^{2}\right)$, and player $i^{3}$ has at least two strategies at his disposal. The function $F^{D^{*},\left(i^{1}, j^{1}\right),\left(i^{2}, j^{2}\right),\left(i^{3}, j^{3}\right)}: \tilde{\Delta} \times \mathbb{C}^{n|D|} \rightarrow \mathbb{C}^{\left|D^{*}\right|} \times \mathbb{C}^{\left|D^{*}\right|}+1$ is specified by the following system of equations:

$$
\begin{aligned}
& u^{i}\left(\delta^{-i}, \tilde{d}^{i}\right)-u^{i}\left(\delta^{-i}, d^{i}\right)=0, \quad\left(d^{i} \in D^{i} \backslash\left\{\tilde{d}^{i}\right\}, i \in N\right), \\
& \sum_{d_{j}^{i} \in D^{i}} \delta_{j}^{i}-1=0, \quad(i \in N), \\
& u^{i}\left(\gamma^{-i}, \tilde{d}^{i}\right)-u^{i}\left(\gamma^{-i}, d^{i}\right)=0, \quad\left(d^{i} \in D^{i} \backslash\left\{\tilde{d}^{i}\right\}, i \in N\right), \\
& \sum_{d_{j}^{i} \in D^{i}} \gamma_{j}^{i}-1=0, \quad(i \in N),
\end{aligned}
$$


(9) $\quad \delta_{j^{3}}^{i^{3}}-\gamma_{j^{3}}^{i^{3}}=0$.

The Jacobian of the function $F^{D^{*},\left(i^{1}, j^{1}\right),\left(i^{2}, j^{2}\right),\left(i^{3}, j^{3}\right)}$ in a point $(\bar{\delta}, \bar{\gamma}, \bar{u}) \in \widetilde{\Delta} \times \mathbb{C}^{n|D|}$ for which it holds that $F^{D^{*},\left(i^{1}, j^{1}\right),\left(i^{2}, j^{2}\right),\left(i^{3}, j^{3}\right)}(\bar{\delta}, \bar{\gamma}, \bar{u})=0$ is depicted in Figure 2, where $E$ stands for a row containing ones only, $e(1)$ is the row with a one in the column belonging to the pair $\left(i^{3}, j^{3}\right)$ and zeros elsewhere, and $e(-1)$ is a row with a -1 in the column belonging to the pair $\left(i^{3}, j^{3}\right)$ and zeros elsewhere. We show that this matrix has full row rank.

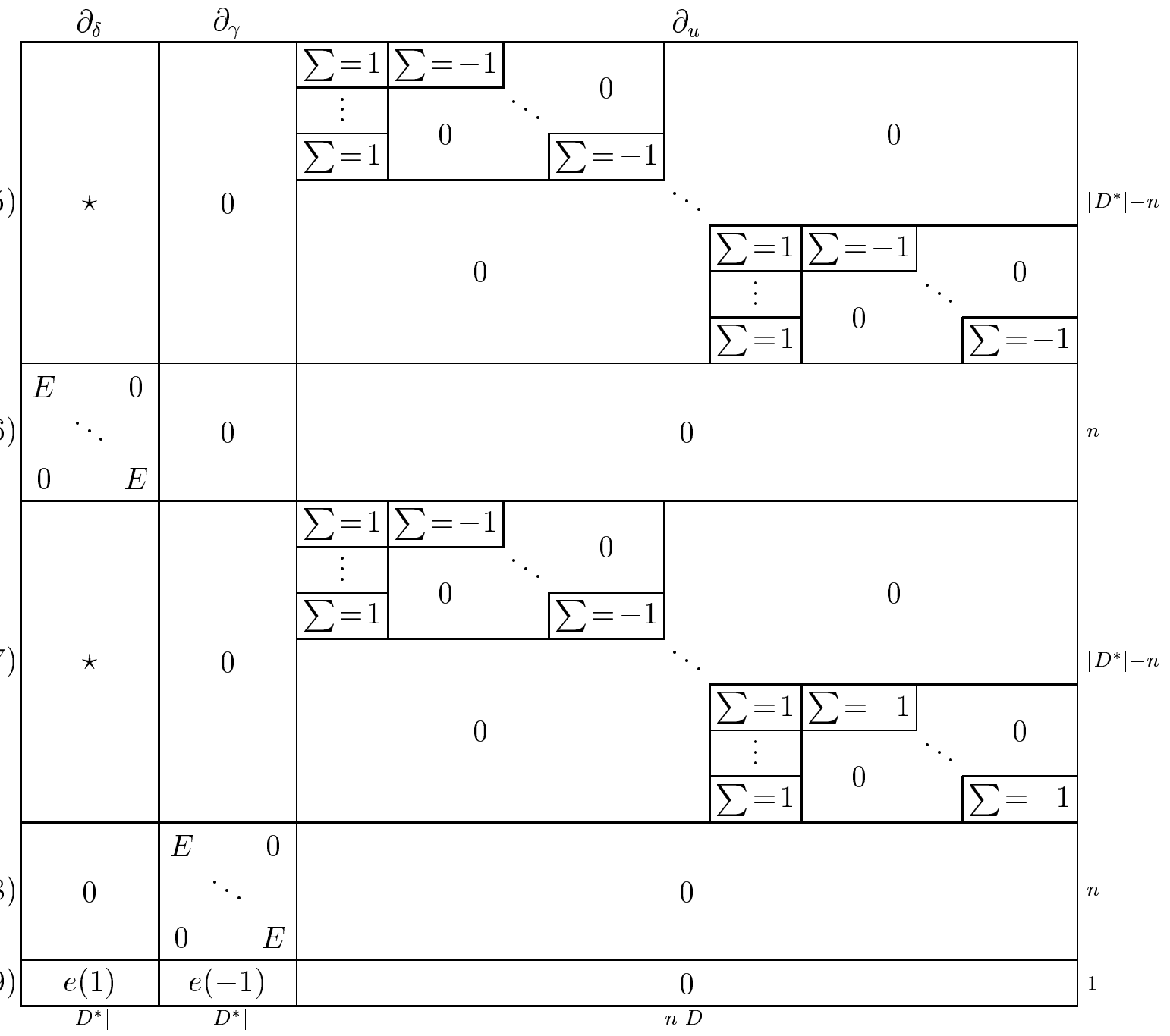

Figure 2: Jacobian of $F^{D^{*},\left(i^{1}, j^{1}\right),\left(i^{2}, j^{2}\right),\left(i^{3}, j^{3}\right)}$.

The derivative with respect to $u$ in (5) has full row rank as has the derivative with respect to $u$ in (7). The compound of the two blocks has full row rank if there does not exist a player $k$ and an action $d_{\ell}^{k} \in D^{k} \backslash\left\{\widetilde{d}^{k}\right\}$ for which the row belonging to $d_{\ell}^{k}$ in $(5)$ depends linearly on the row belonging to $d_{\ell}^{k}$ in (7). Because for both rows the sum of the 
elements equals 1 , the two rows belonging to $d_{\ell}^{k}$ can only be linearly dependent if they are identical.

If $k=i^{1}$, then at least one of the partial derivatives with respect to $u^{k}\left(d_{\ell}^{k}, d_{j^{2}}^{i^{2}}, d^{-i^{2}, k}\right)$ differs in (5) and (7), since $\delta_{j^{2}}^{i^{2}} \neq \gamma_{j^{2}}^{i^{2}}$. If $k \neq i^{1}$, then at least one of the partial derivatives with respect to $u^{k}\left(d_{\ell}^{k}, d_{j^{1}}^{i^{1}}, d^{-i^{1}, k}\right)$ differs in (5) and (7), since $\delta_{j^{1}}^{i^{1}} \neq \gamma_{j^{1}}^{i^{1}}$. In both cases it holds that the rows in (5) and (7) belonging to $d_{\ell}^{k}$ are different. As this holds for all pairs $(k, \ell) \in D^{k} \backslash\left\{\tilde{d}^{k}\right\}$ and all $k \in N$, it follows that the derivative with respect to $u$ in $(5)$ and (7) has full row rank. Since, the derivatives with respect to $u$ in (6), (8) and (9) are all zero, it is sufficient to show that the matrix of partial derivatives to $\delta$ and $\gamma$ in (6), (8) and (9) has full row rank.

It is easily seen that the matrix of partial derivatives with respect to $\delta$ and $\gamma$ in (6) and (8) has full row rank. The only thing left is to show that the row in (9) does not linearly depend on the rows in (6) and (8). Note that row (9) can only depend linearly on (6) and (8) if $D^{i^{3}}=\left\{d_{j^{3}}^{i^{3}}\right\}$. This is ruled out, since player $i^{3}$ was chosen to have at least two pure strategies at his disposal.

Since the Jacobian of $F^{D^{*},\left(i^{1}, j^{1}\right),\left(i^{2}, j^{2}\right),\left(i^{3}, j^{3}\right)}$ evaluated at $(\bar{\delta}, \bar{\gamma}, \bar{u})$ has full rank for all $(\bar{\delta}, \bar{\gamma}, \bar{u})$ such that $F^{D^{*},\left(i^{1}, j^{1}\right),\left(i^{2}, j^{2}\right),\left(i^{3}, j^{3}\right)}(\bar{\delta}, \bar{\gamma}, \bar{u})=0$, it follows by the transversality theorem (see, for example, Mas-Colell (1985), Theorem I.2.2) that $\partial_{(\delta, \gamma)} F^{D^{*},\left(i^{1}, j^{1}\right),\left(i^{2}, j^{2}\right),\left(i^{3}, j^{3}\right)}(\bar{\delta}, \bar{\gamma})$ has full row rank for all $(\bar{\delta}, \bar{\gamma})$ such that $F^{D^{*}, u}(\bar{\delta}, \bar{\gamma})=0$, except for a set of vectors $u$ with zero Lebesgue measure. Denote the set of vectors $u$ for which the full row rank property holds by $\mathcal{U}\left(D^{*},\left(i^{1}, j^{1}\right),\left(i^{2}, j^{2}\right),\left(i^{3}, j^{3}\right)\right)$. By counting equations and unknowns, it follows that $\partial_{(\delta, \gamma)} F^{D^{*},\left(i^{1}, j^{1}\right),\left(i^{2}, j^{2}\right),\left(i^{3}, j^{3}\right)}(\bar{\delta}, \bar{\gamma})$ cannot have full row rank. It follows that for all $u \in \mathcal{U}\left(D^{*},\left(i^{1}, j^{1}\right),\left(i^{2}, j^{2}\right),\left(i^{3}, j^{3}\right)\right)$ there are no solutions to $F^{D^{*},\left(i^{1}, j^{1}\right),\left(i^{2}, j^{2}\right),\left(i^{3}, j^{3}\right), u}(\bar{\delta}, \bar{\gamma})=0$.

Define the full measure set $\mathcal{U}$ as the intersection of all sets $\mathcal{U}\left(D^{*},\left(i^{1}, j^{1}\right),\left(i^{2}, j^{2}\right),\left(i^{3}, j^{3}\right)\right)$ and the set of vectors $u$ given in Theorem 3 . Fix a vector $u$ in $\mathcal{U}$. We show that for the game generated by $u$, there is no $D^{*}$ such that $F^{D^{*}, u}$ has multiple solutions with the properties as in the first paragraph of the proof.

Suppose there are multiple solutions, $\delta$ and $\gamma$, in which a single player $i^{3}$ with at least two strategies at his disposal plays action $d_{j^{3}}^{i}$ with the same probability in both candidate equilibria, i.e. $\delta_{j^{3}}^{i^{3}}=\gamma_{j^{3}}^{i^{3}}$.

Suppose first that for some player $i \in N, \delta^{-i}=\gamma^{-i}$. Then it follows from the multilinearity of $F^{D^{*}, u}$ that for all linear combinations $\rho^{i}$ of $\delta^{i}$ and $\gamma^{i}$, the point $\left(\rho^{i}, \delta^{-i}\right)=\left(\rho^{i}, \gamma^{-i}\right)$ is a zero of $F^{D^{*}, u}$. So, we have a continuum of zeros, which contradicts the game coming from the generic set of Theorem 3. As a consequence, it is not possible that $\delta^{-i}=\gamma^{-i}$ for any $i \in N$. We conclude that there are two different players $i^{1}$ and $i^{2}$, a strategy $j^{1}$ for player $i^{1}$, and a strategy $j^{2}$ for player $i^{2}$ such that $\delta_{j^{1}}^{i^{1}} \neq \gamma_{j^{1}}^{i^{1}}$ and $\delta_{j^{2}}^{i^{2}} \neq \gamma_{j^{2}}^{i^{2}}$. Since 
$\delta_{j^{3}}^{i^{3}}=\gamma_{j^{3}}^{i^{3}}$, there is a solution to $F^{D^{*},\left(i^{1}, j^{1}\right),\left(i^{2}, j^{2}\right),\left(i^{3}, j^{3}\right), u}(\bar{\delta}, \bar{\gamma})=0$. Since $u \in \mathcal{U}$, this leads to a contradiction. This completes our proof.

\section{References}

Adams, W.W., and P. Loustaunau (1994), An Introduction to Gröbner Bases, Graduate Studies in Mathematics, Volume 3, American Mathematical Society, Providence.

Allgower, E.L., And K. Georg (1980), "Simplicial and Continuation Methods for Approximating Fixed Points and Solutions to Systems of Equations," SIAM Review, 22, 28-85.

Allgower, E.L., And K. Georg (1983), "Predictor-Corrector and Simplicial Methods for Approximating Fixed Points and Zero Points of Nonlinear Mappings," in A. Bachem, M. Grötschel, and B. Korte (eds.), Mathematical Programming: The State of the Art, Springer-Verlag, Berlin, Heidelberg, New York, pp. 15-56.

Allgower, E.L., And K. Georg (1990), Numerical Continuation Methods: An Introduction, Springer-Verlag, Berlin, Heidelberg.

Allgower, E.L., And K. Georg (1993), "Continuation and Path Following," Acta Numerica, 1-64.

Bochnak, J., M. Coste, And M-F. Roy (1987), Géométrie Algébrique Réelle, SpringerVerlag, Berlin.

Borm, P., A. Gijsberts, And S.H. TiJs (1989), "A Geometric-Combinatorial Approach to Bimatrix Games," Methods of Operations Research, 59, 199-209.

Bubelis, V. (1979), "On Equilibria in Finite Games," International Journal of Game Theory, 8, 65-79.

Buchberger, B. (1965), Ein Algorithmus zum Auffinden der Basiselemente des Restklassenringes nach einem nulldimensionalen Polynomideal, $\mathrm{Ph}$. D. Thesis, University of Innsbruck, Innsbruck, Austria.

Chow, S.N., J. Mallet-Paret, and J.A. Yorke (1979), "A Homotopy Method for Locating All Zeros of a System of Polynomials," in H.O. Peitgen, and H.O. Walther (eds.), Functional Differential Equations and Approximation of Fixed Points, 730, Springer-Verlag, Berlin, Heidelberg, New York, pp. 228-237.

Cox, D.A., J.B. Little, And D. O'SheA (1996), Ideals, Varieties, and Algorithms: An Introduction to Computational Algebraic Geometry and Commutative Algebra, Undergraduate Texts in Mathematics, Springer-Verlag, New York. 
DAtTA, R.S. (2002), "University of Nash Equilibria," mimeo.

Dickhaut, J., And T. Kaplan (1993), "A Program for Finding Nash Equilibria," in H.R. Varian (ed.), Economic and Financial Modeling with Mathematica, Springer-Verlag, New York.

Drexler, F.J. (1977), "Eine Methode zur Berechnung sämtlicher Lösungen van Polynomgleichungssystemen," Numerische Mathematik, 29, 45-58.

Drexler, F.J. (1978), "A Homotopy-Method for the Calculation of All Zero-Dimensional Polynomial Ideals," in H.G. Wacker (ed.), Continuation Methods, Academic Press, New York, pp. 69-93.

Garcia, C.B., And T.Y. Li (1980), "On the Number of Solutions to Polynomial Systems of Equations," SIAM Journal on Numerical Analysis, 17, 540-546.

Garcia, C.B., And W.I. Zangwill (1979a), "Finding All Solutions to Polynomial Systems and Other Systems of Equations," Mathematical Programming, 16, 159-176.

Garcia, C.B., And W.I. Zangwill (1979b), "Determining All Solution to Certain Systems of Nonlinear Equations," Mathematics of Operations Research, 4, 1-14.

Garcia, C.B., And W.I. Zangwill (1980), "Global Continuation Methods for Finding All Solutions to Polynomial Systems of Equations in $N$ Variables," in A.V. Fiacco, and K.O. Kortanek (eds.), Extremal Methods and Systems Analysis, Springer-Verlag, Berlin, Heidelberg, New York, pp. 481-497.

Garcia, C.B., And W.I. Zangwill (1981), Pathways to Solutions, Fixed Points, and Equilibria, Pentice-Hall Inc., Englewood Cliffs, New Jersey.

GröBner, W. (1949), Moderne Algebraische Geometrie, Die Idealtheoretischen Grundlagen, Springer-Verlag, Wien, Innsbruck.

GröBner, W. (1970), Algebraische Geometrie II, Bibliografisches Institut, Mannheim.

Harsanyi, J.C. (1973), "Oddness of the Number of Equilibrium Points," International Journal of Game Theory, 2, 235-250.

Herings, P.J.J. (1997), "A Globally and Universally Stable Price Adjustment Process," Journal of Mathematical Economics, 27, No. 2, 163-193.

Herings, P.J.J., And R. Peeters (2001), "A Differentiable Homotopy to Compute Nash Equilibria of n-Person Games," Economic Theory, 18, 159-186. 
Jongen, H.T., P. Jonker, And F. Twilt (1983), Nonlinear Optimization in $\mathbb{R}^{n}$, I. Morse Theory, Chebyshev Approximation, Methoden und Verfahren der mathematische Physik, 29, Peter Lang, Frankfurt.

Kojima, M., And S. Mizuno (1983), "Computation of All Solutions to a System of Polynomial Equations," Mathematical Programming, 25, 131-157.

Kostreva, M.M., and L.A. Kinard (1991), "A Differential Homotopy Approach for Solving Polynomial Optimization Problems and Noncooperative Games," Computers and Mathematics with Applications, 21, 135-143.

Mas-Colell, A. (1985), The Theory of General Economic Equilibrium: A Differentiable Approach, Cambridge University Press, Cambridge.

McKelvey, R.D. (1996), "A Liapunov Function for Nash Equilibria," California Institute of Technology, Social Science Working Paper \#953.

McKelvey, R., And A. McLennan (1996), "Computation of Equilibria in Finite Games," in H.M. Amman, D.A. Kendrick, and J. Rust (eds.), Handbook of Computational Economics, Volume I, Elsevier Science, Amsterdam, pp. 87-142.

McKelvey, R.D., And A. McLennan (1997), "The Maximal Number of Regular Totally Mixed Nash Equilibria," Journal of Economic Theory, 72, 411-425.

McLennan, A. (1997), "The Maximal Generic Number of Pure Nash Equilibria," Journal of Economic Theory, 72, 408-410.

McLennan, A. (1999), "The Expected Number of Nash Equilibria of a Normal Form Game," mimeo.

Mizuno, S. (1981), "A Simplicial Algorithm for Finding All Solutions to Polynomial Systems of Equations," Research Reports B-97, Department of Information Sciences, Tokyo Institute of Technology, Tokyo.

Morgan, A.P. (1983), "A Method for Computing All Solutions to Systems of Polynomial Equations," ACM Transactions on Mathematical Software, 9, 1-17.

Morgan, A.P. (1987), Solving Polynomial Systems Using Continuation for Engineering and Scientific Problems, Prentice-Hall Inc., Upper Saddle River, New Jersey.

Morgan, A.P., And A.J. Sommese (1987), "Computing All Solutions to Polynomial Systems Using Homotopy Continuation," Applied Mathematics and Computation, 24, 115138. 
Morgan, A.P., A.J. Sommese, and L.T. Watson (1989), "Finding All Isolated Solutions to Polynomial Systems Using HomPACK," ACM Transactions on Mathematical Software, 15, 93-122.

Rosenmüller, J. (1971), "On a Generalization of the Lemke-Howson Algorithm to Noncooperative N-Person Games," SIAM Journal on Applied Mathematics, 21, 73-79.

Watson, L.T., S.C. Billups, and A.P. Morgan (1987), "HOMPACK: A Suite of Codes for Globally Convergent Homotopy Algorithms," ACM Transactions on Mathematical Software, 13, 281-310.

Wilson, R. (1971), "Computing Equilibria of $n$-Person Games," SIAM Journal of Applied Mathematics, 21, 80-87.

Wright, A.H. (1985), "Finding All Solutions to a System of Polynomial Equations," Mathematics of Computation, 44, 125-133.

Zulehner, W. (1988), "A Simple Homotopy Method for Determining All Isolated Solutions to Polynomial Systems," Mathematics of Computation, 50, 167-177. 